\title{
抗肿瘤血管生成免疫治疗的研究进展 ${ }^{*}$
}

\section{侯建梅 田 聆 魏于全 ${ }^{* *}$}

(四川大学华西医院人类疾病生物治疗重点实验室 肿瘤中心, 成都 610041)

\begin{abstract}
摘要 肿瘤的生长和转移依赖血管生成, 近年来抗血管生成治疗肿瘤已取得了较大的进展, 在 被动免疫治疗方面, 重组的人源化抗血管内皮生长因子单克隆抗体 Avastin 已正式被美国食品及 药物管理局批准为抗肿瘤血管生成药物, 这是迄今第一个抗肿瘤血管生成的生物制剂; 在主动免 疫治疗方面，通过多种途径如异种同源分子的交叉免疫反应或树突状细胞的抗原递呈功能等成 功突破了机体对自身抗原的免疫耐受, 产生了较好的抗肿瘤血管生成作用. 本文就近年来抗肿瘤 血管生成免疫治疗方面的研究和进展做一综合论述.
\end{abstract}

\section{关键词 血管生成 肿瘤 免疫治疗}

自 20 世纪 70 年代初期Harvard大学J. Folkman教 授提出实体肿瘤的生长和转移依赖于新生血管生成 这一观点以来, 许多研究者对这一理论进行了广泛 深入的研究, 并形成了抗肿瘤血管生成疗法 ${ }^{[1,2]}$. 传 统的肿瘤治疗多针对肿瘤细胞, 而抗肿瘤血管生成 治疗是通过抑制或破坏肿瘤血管生成, 切断肿瘤赖 以生存的“生命线”, “饿死”肿瘤. 与传统的肿瘤治疗 相比较, 抗肿瘤血管生成策略有较多的优势, 表现在: （i ）受肿瘤耐药性影响小, 因为供应肿瘤营养的血 管内皮细胞是遗传稳定的正常二倍体细胞, 即使多 次反复给药甚至肿瘤复发再给药也不易产生耐药性; （ii）药物易于到达血管靶细胞，无需进入肿瘤细胞 内部或通过血脑屏障; (iii) 由于各种肿瘤血管内皮 细胞基本相同, 不同肿瘤抗血管生成治疗的研究经
验可以互相推广, 而无须考虑肿瘤的组织学特性; (iv) 对肿瘤转移有抑制作用, 因为肿瘤转移过程和血管 生成密切相关，抑制血管生成则可抑制肿瘤转移; ( v ) 作用较强, 有根治肿瘤的可能, 由于旁观者效应或下 游放大效应, 有限数量的内皮细胞的破坏能够引起 肿瘤组织大范围的坏死和凋亡; ( vi ) 抗肿瘤血管生 成较安全, 不良反应较小. 在正常的成年人中, 除了 创伤愈合以及生殖周期外，几乎所有的新生血管都 是病理性的, 如肿瘤、风湿性关节炎、银屑病及视网 膜病变等 $[3 \sim 6]$. 因此, 以血管为靶向的抗血管生成有 望成为治疗肿瘤的新疗法.

血管生成受血管生长刺激因子和抑制因子等因 素的调控, 目前已证实的促血管生成因子主要有: 血 管内皮生长因子(vascular endothelial growth factor, 
VEGF)、血小板衍生生长因子(platelet-derived growth factor, PDGF)、碱性成纤维细胞生长因子(basic fibroblast growth factor, bFGF)、转化生长因子(transfoming growth factor, TGF)、胰岛素样生长因子(insulinlike growth factor, IGF)、表皮生长因子等(epidermal growth factor, EGF), 而抑制因子主要有血管抑制素, 内皮抑素，凝血栓蛋白-1, 血小板因子-4(platelet factor-4, PF-4)，基质金属蛋白酶抑制剂，生长抑素等. 这些血管生长刺激因子和抑制因子通过自分泌或旁 分泌的方式作用于血管内皮细胞, 影响其增殖、迁移 和形成管状结构, 二者间的平衡决定了血管的生成 或抑制. 肿瘤的发生和发展, 通常是抑制血管生成的 生长因子失活或缺失、促进血管生成的调节因子占 主导地位并作用于内皮细胞的结果 ${ }^{[7]}$.

目前抗肿瘤血管生成的各种研究主要从两个方
向着手，其一，增加血管抑制调节因子，主要通过重 组蛋白或基因治疗的方式补充内皮抑素、血管抑制 素、基质金属蛋白酶组织抑制剂等. 其二, 减少或抑 制促血管生成调节因子，一方面，通过多种单克隆抗 体(如 VEGF 单克隆抗体 Avastin, EGFR 单克隆抗体 Ebitux, VEGFR-2 单克隆抗体 IMC-1C-11 等)阻断封 闭生长因子信号传导; 另一方面通过主动免疫的方 法诱导机体产生针对于血管生成相关的靶细胞或靶 分子的免疫反应(图 1); 此外，一些人工合成的小分 子化合物如已被美国食品和药物管理局正式批准的 EGFR 抑制剂 ZD1839(Iressa)、已进入临床 I / II 期试 验的血管内皮细胞酪氨酸激酶受体抑制剂 SU5416, MMP 抑制剂 Neovastat, 烟曲霉素衍生物 TNP470 等 都显示了较好的抗肿瘤血管生成作用. 本文主要就 近年来抗肿瘤血管生成免疫治疗方面的研究进展综

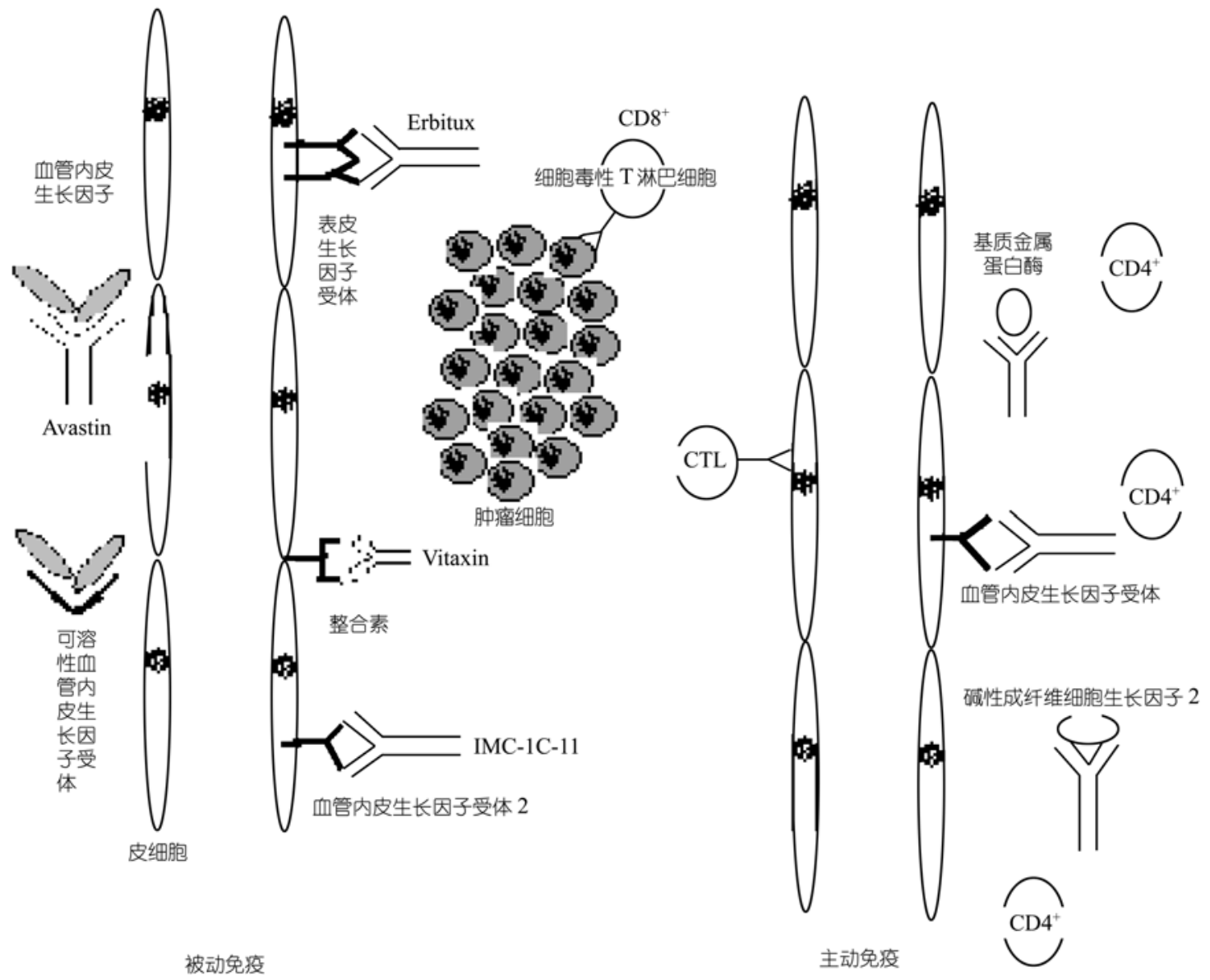

图 1 抗肿瘤血管形成免疫治疗的途径 
述如下.

\section{1 被动免疫治疗}

\section{1 中和促血管生成因子}

众所周知, 抗体具有中和作用, 可利用抗血管生 成促进因子的抗体中和相应的生长因子, 阻断随后的 信号通路，从而抑制肿瘤血管生成. Kim等人 ${ }^{[8]}$ 使用 VEGF特异的单克隆抗体治疗接种人横纹肌肉瘤、多 型性胶质母细胞瘤或平滑肌肉瘤模型的裸鼠, 可显著 抑制肿瘤的生长, 肿瘤组织内血管生成也受到明显抑 制, 微血管密度减少, 血管通透性降低, 血管直径减 小, 肿瘤播散性转移以及恶性腹水的形成均受到抑制. 目前重组的人源化抗 VEGF单克隆抗体 Bevacizumab(商品名为 Avastin $^{\mathrm{TM}}$ ) 已正式被美国食品 及药物管理局(FDA)批准为抗血管生成治疗用药, 其 中, Avastin联合IFL(5-氟尿嘧啶/甲酰四氢叶酸/依利替 康)化疗方案已成为转移性结肠癌的一线治疗方案.

此外, 利用生长因子与其相应受体之间高亲和 力结合的性质, 使用可溶性受体也可起到中和生长因 子的作用. Takayama等人 ${ }^{[9]}$ 构建了可溶性人VEGFR-1 胞外段重组腺病毒, 将其转染肿瘤细胞后发现, 肿瘤 细胞在小鼠体内的成瘤性显著降低, 且肿瘤组织内血 管生成受到明显抑制, 调亡增多, 并证实这种效应是 转染细胞分泌到体内的可溶性VEGFR与VEGF结合 使其功能失活引起的, 并且可溶性VEGFR还可能与 野生型VEGFR结合形成异二聚体阻断其信号传导. 促 血管生成素-1 通过与一种血管内皮细胞特异的、具有 酪氨酸激酶活性受体Tie-2 的结合, 在血管生成的过 程中发挥重要作用. Lin等人 ${ }^{[10]}$ 构建了以腺病毒为表达 载体的重组、可溶性的Tie-2(AdExTek), 研究显示可 溶性的Tie-2(ExTek)能够竞争性地与促血管生成素-1 结合, 阻断了促血管生成素-1 介导的Tie-2 的磷酸化作 用，抑制了肿瘤血管生成，进而抑制了肿瘤生长.

\section{2 封闭促血管生成生长因子受体}

血管内皮生长因子(VEGF) 是目前发现的最为强 大、专一的刺激血管内皮细胞增生的因子, VEGF选择 性直接作用于血管内皮细胞膜上的两种 III 型酪氨酸 激酶受体 (VEGFR-1/Flt-1 和 VEGFR-2/KDR/Flk-1).
VEGFR-2 是血管生成的主要调控分子，具有明显的 化学趋化和促分裂作用, 与血岛、血管生成和造血有 关, 而VEGFR-1 主要在内皮细胞排列形成管腔时发 挥作用. 它们之间高效特异地结合, 刺激血管发生和 生长，同时增加血管通透性，促进血管内血浆蛋白等 物质外渗, 为血管内皮细胞地迁移、毛细血管网的形 成提供了良好基质 ${ }^{[11]}$. 利用抗体封闭生长因子受体, 可阻断受体/配体间信号传导. 与抗VEGF抗体相比, 抗VEGFR的抗体能够特异性地靶向血管内皮细胞上 过表达的VEGFR，而无需中和由肿瘤组织产生的大 量的VEGF. Prewett等人 ${ }^{[12]}$ 报道抗小鼠VEGFR-2 的 单克隆抗体mAb DC101 经腹腔内给药能够抑制藻酸 盐包裹肿瘤细胞实验以及Matrigel plug实验中的血管 生成，并且对多种小鼠肿瘤以及人肿瘤的裸鼠模型产 生了抑制作用。目前抗 VEGFR-2(KDR) 抗体 IMC-1C11 已进入结肠癌治疗的 I 期临床试验. Niederman等人 ${ }^{[13]}$ 利用 $T$ 细胞受体嵌合技术制备了一种重 组的逆转录病毒载体，该载体表达一种由VEGF序列 和 $\mathrm{T}$ 细胞受体链胞内信号传导序列共同组成的嵌合 性 $\mathrm{T}$ 细胞受体. 将这种重组逆转录病毒转染小鼠 $\mathrm{CD} 8^{+}$ $\mathrm{T}$ 淋巴细胞后, 转染细胞在体外实验显示具有特异性 杀伤表达VEGFR细胞的活性. 这种基因修饰后的细 胞毒性T淋巴细胞过继性输注至荷瘤小鼠体内能够显 著抑制一系列同源小鼠肿瘤和人肿瘤移植模型的生 长. 该项研究为以VEGF/VEGFR为靶向的抗肿瘤血 管生成治疗提供了一个新的方向.

表皮生长因子受体(EGFR)是CerbB1/EGFR 基因 编码的Src族跨膜受体酪氨酸激酶, 由胞外段、跨膜段 及胞内激酶活性段构成, 其胞外段的主要功能是结合 表皮生长因子 $(\mathrm{EGF}) 、$ 肿瘤生长因子 $\alpha(\mathrm{TGF} \alpha)$ 等配体介 导受体同源二聚化或异源二聚化, 进而启动EGFR通 路信号转导. 异常活化的EGFR通路可改变细胞的生 长特性, 以一种EGF等配体依赖的方式促进细胞的恶 性转化以及肿瘤的发生. 而既成肿瘤也可以自分泌或 旁分泌的形式产生EGF, TGF $\alpha$ 等特异配体，促进肿瘤 细胞的增殖、粘附、转移以及血管生成, 抑制肿瘤细 胞的凋亡, 从而促进EGFR高表达肿瘤的进展 ${ }^{[14,15]}$. 近年来, 多项研究显示抗EGFR单克隆抗体能够高亲 和力地与EGFR结合, 抑制受体酪氨酸激酶的活性, 
通过下调促血管生成因子如 VEGF, bFGF, IL-8, MMP-9, 诱导肿瘤细胞以及内皮细胞的调亡, 从而抑 制肿瘤诱导的血管生成, 抑制肿瘤生长 ${ }^{[16]}$. 其中, ImClone 公司研发的抗 EGFR 单克隆抗体 Cetuximab(C225, Erbitux $\left.{ }^{\mathrm{TM}}\right)$ 已被美国FDA批准为治疗 EGFR阳性结肠癌的药物, 它可用于对以依利替康为 基础的化疗方案无效或耐受的转移性结肠癌患者.

此外, 新生血管生成依赖于内皮细胞的粘附和 游出. 整合素是一类由 $\alpha$ 亚基和 $\beta$ 亚基构成的异二聚 体跨膜蛋白, 它们通过与细胞外基质分子的相互作用 而控制细胞的移动、分化和增殖. 在肿瘤血管生成的 过程中, 整合素 $\alpha v \beta 3$ 以及 $\alpha v \beta 5$ 在增生的血管内皮细 胞表面表达水平增高. 其中, bFGF促血管生成作用需 要 $\alpha v \beta 3$ 参与, 而 VEGF诱导的血管生成过程需要 $\alpha v \beta 5$ 的参与, 有研究表明基质金属蛋白酶-2 (MMP-2) 通过与 $\alpha v \beta 3$ 的直接结合有助于血管内皮细胞增生处 胶原酶活性的升高 ${ }^{[17 ~ 19]}$. 上述发现使得整合素 $\alpha v \beta 3$ 在抗血管生成中成为一个重要的靶分子. Vitaxin是一 种通过噬菌体展示技术获得的人源化的小鼠单克隆 抗体LM609, 它能够直接与人整合素 $\alpha v \beta 3$ 结合, 诱 导内皮细胞凋亡，抑制肿瘤新生血管生成，目前已进 入临床 11 期试验 20$]$.

血管内皮细胞之间通过类似于上皮细胞之间的 紧密连接形成一个整体。血管内皮细胞钙粘素 (VE-cadherin)是一种内皮细胞特异的跨膜蛋白，在血 管生成过程中对血管形态构成发挥重要作用 ${ }^{[21]}$. 研 究表明, 使用血管内皮细胞钲粘素单克隆抗体 $\mathrm{mAb}$ BV13 以及 $m A b$ BV14 均可阻断VE-cadherin的黏附特 性，抑制血管生成和肿瘤生长. 但mAb BV13 在抑制 血管生成的同时，体内肺脏以及心脏组织的血管通透 性显著升高, 而 $\mathrm{mAb}$ BV14 则对血管通透性没有明显 影响. 我们认为这是由于两种抗体与VE-cadhe- rin结 合的部位不同引起的 ${ }^{[22,23]}$.

\section{2 主动免疫治疗}

以肿瘤抗原为基础的肿瘤疫苗能够主动特异地 激发机体对肿瘤的免疫应答, 然而, 到目前为止, 除 了少数肿瘤如黑色素瘤、前列腺癌具有较特异的肿 瘤抗原外，多数肿瘤缺乏特异性抗原; 同时，已鉴定
的肿瘤相关抗原多数是自身分子，一般情况下，机体 免疫系统对自身分子呈免疫耐受，难以诱导有效的免 疫反应 ${ }^{[24]}$. 因此, 如何打破机体对与肿瘤生长以及血 管生成相关分子的免疫耐受，激发有效的抗肿瘤免疫 反应成为研究热点, 目前的研究主要集中在以下几个 方向.

\section{1 以异种同源分子免疫交叉反应为基础的抗肿 瘤血管生成免疫治疗}

大量的研究发现, 许多自身免疫性疾病因为微 生物的感染而诱发或加重, 病原微生物的抗原与自身 免疫性疾病靶分子的相似性在自身免性疾病发生和 进展中起着重要的作用 ${ }^{[25]}$, 那么, 能否用一定相似性 的抗原来诱导机体产生针对自身分子的免疫反应? 许多分子在生物进化过程中相对保守，它们既要保持 序列的稳定性以维持相似的生理功能, 又要有一定的 进化以适应不同的环境和生理需要, 这就形成了生物 进化过程中的不同种属之间的异种同源分子. 近年来, 我们针对与血管生成相关的分子或细胞, 利用其在小 鼠和其他种属之间的异种同源性做了一系列研究 [26,27].

肿瘤组织中新生血管内皮细胞表面表达一些在 正常血管内皮细胞上没有或几乎检测不到的蛋白分 子，如 $\alpha v \beta 3$ 整合素以及一些生长因子的受体，小鼠 血管内皮细胞上的这些蛋白质分子与人类及其他种 属中相应的分子具有不同程度的同源性. 我们用经甲 醛固定处理的异种(人、牛)血管内皮细胞作为疫苗免 疫小鼠，利用其异种同源性成功地打破了小鼠体内对 自身血管内皮细胞的免疫耐受，产生了保护性及治疗 性的抗肿瘤免疫反应. 实验中观察到纯化的经异种疫 苗免疫的小鼠体内的免疫球蛋白在体外能够抑制内 皮细胞增殖，过继性实验显示纯化的自身反应性免疫 球蛋白能够抑制肿瘤血管生成，同时小鼠肿瘤生长受 到抑制; 免疫组化染色发现经过异种疫苗免疫的小 鼠的肿瘤组织中的血管内皮细胞上有自身抗体沉积; Western blot检测发现，小鼠内皮细胞提取物与经过 异种疫苗免疫后的小鼠血清反应出现多个阳性条带, 其中至少有两条带的分子量 (分别为 220 和 $130 \mathrm{kD}$ )与 已知的血管内皮细胞生长因子受体 2 以及整合素 $\alpha \mathrm{v}$ 
的分子量相近. 研究表明, 异种血 管内皮细胞疫苗 抗肿瘤作用的产生和诱导抗小鼠血管内皮细胞自身 抗体的产生与体内 $\mathrm{CD} 4^{+}$淋巴细胞有关 ${ }^{[27]}$.

基于此，我们利用异种分子的同源性针对性地 选择了与肿瘤血管发生有关的几个重要靶分子进行 研究. 序列比较分析发现, 非洲爪蟾的VEGF在氨基 酸序列上与小鼠VEGF164 及人VEGF165 分别有 $75 \%$ 和 $73 \%$ 的同源性. 我们分别构建小鼠和非洲爪蟾 VEGF的重组真核表达质粒，分别命名为MVEGF-p及 XVEGF-p, 并由此制备核酸疫苗. XVEGF-p免疫小鼠 在多个肿瘤模型中产生了保护性及治疗性的抗肿瘤 免疫效应, Western blot和 ELISA 检测都发现在 XVEGF-p免疫小鼠的血清中有抗VEGF特异性抗体存 在, 纯化的免疫球蛋白在体外能够抑制由VEGF介导 的内皮细胞的增殖，体内过继性实验能够抑制肿瘤血 管生成，产生抗肿瘤效应. 经XVEGF-p免疫的荷瘤小 鼠体内的VEGF水平低于对照组, 抗CD4 单抗能阻断 血清中免疫球蛋白 $\operatorname{IgG} 1$ 和 $I g G 2 b$ 的升高, 同时也阻断 了异种VEGF核酸疫苗的抑瘤作用 ${ }^{[28]}$. 此后, 我们相 继构建了非洲爪蟾VEGF重组蛋白质疫苗、非洲爪蟾 FGFR 1 核酸疫苗、鸡整合素 $\beta_{3}$ 核酸疫苗、鸡基质金属 蛋白酶 2(MMP-2)核酸疫苗、䣫鹑VEGFR-2 重组蛋白 质疫苗、鸡EGFR核酸疫苗以及蛋白质疫苗等，研究 显示上述疫苗均成功打破了机体对自身分子的免疫 耐受，产生了针对靶向分子特异性的自身抗体，阻断 了其在肿瘤血管生成中相应的作用, 抑制了血管生成, 产生了依赖于 $\mathrm{CD}^{+}{ }^{+} \mathrm{T}$ 淋巴细胞的抗肿瘤效应 ${ }^{[28 \sim 32]}$

通常认为 $\mathrm{CD} 8^{+}$淋巴细胞介导的细胞毒作用在抗 肿瘤免疫效应中占主要地位，但近年来 $\mathrm{CD}^{+}$淋巴细 胞在抗肿瘤免疫反应中的作用也得到了越来越多研 究者的重视. 有研究者发现在小鼠黑色素瘤模型中, $\mathrm{CD} 4^{+}$淋巴细胞亚群是抗肿瘤免疫所必需的, 去除 $\mathrm{CD}^{+}$淋巴细胞抗肿瘤免疫应答就不会产生, 且 $\mathrm{CD} 4^{+}$ 淋巴细胞对于激发和维持免疫记忆是必不可少的 ${ }^{[33]}$. 还有研究表明 $\mathrm{CD}^{+}{ }^{+}$淋巴细胞甚至可以单独对某些 MHC II 类分子阴性的肿瘤细胞产生杀伤作用 ${ }^{[34]}$. 目 前已知 $\mathrm{CD}^{+}{ }^{+}$淋巴细胞通过分泌细胞因子和表达表面 分子而调控和放大免疫应答，在抗体依赖的免疫过程 中, 其在免疫阶段和效应阶段均需要 $\mathrm{CD} 4{ }^{+}$淋巴细胞
的参与 ${ }^{[35]}$. 在自身免疫性疾病(如变应性脑炎、系统性 红斑狼疮、自身免疫性胃炎等)中, $\mathrm{CD}^{+}$淋巴细 胞的 作用同样扮演了重要角色 ${ }^{[36,37]}$, 这些研究结果有助于 我们理解 $\mathrm{CD} 4^{+}$淋巴细胞在异种同源分子诱导自身免 疫性反应过程中所起的作用.

\section{2 非异种同源分子交叉免疫反应机制打破肿瘤 血管生成相关分子免疫耐受的抗肿瘤血管生成免 疫治疗}

肿瘤免疫逃逸是肿瘤恶性生长的主要原因之一, 产生肿瘤免疫耐受的因素较多且复杂, 其中肿瘤细胞 免疫原性弱或肿瘤相关抗原不能有效递呈给 $\mathrm{T}$ 细胞产 生特异性的免疫反应在肿瘤生长过程中起一定作用 因此，树突状细胞(DC)的抗原提呈功能对于诱导抗 肿瘤血管生成的靶向免疫治疗具有积极意义. Li等人 [38]使用可溶性的VEGFR-2(Flk-1)蛋白刺激DC，并免 疫小鼠，小鼠体内产生了特异性抗Flk-1 抗体以及 $\mathrm{CD}^{+}$细胞毒性 $\mathrm{T}$ 细胞反应, 藻酸盐实验显示肿瘤诱导 的血管生成受到抑制，小鼠体内肿瘤生长也受到了显 著抑制. Nair等人 ${ }^{[39]}$ 将编码VEGFR-2, VEGF的质粒转 染DC后免疫小鼠产生了保护性和治疗性的抗肿瘤免 疫反应，小鼠体内产生了细胞毒性 $\mathrm{T}$ 淋巴细胞(CTL) 反应, 体外 $\mathrm{C}_{\mathrm{r}} 51$ 释放实验检测CTL活性显示, 在接受 转染了VEGFR-2, VEGF的 DC细胞免疫后, 小鼠体 内的淋巴细胞能够特异识别转染了 VEGFR-2, VEGF 的BLK.SV肿瘤细胞，并产生细胞毒性作用。实验中 肿瘤的血管生成受到了显著抑制. 该项研究同时显示, 将上述抗血管生成的免疫治疗与以肿瘤细胞相关抗 原(端粒酶)为靶向的免疫治疗相结合能够产生协同增 强的抗肿瘤作用. 这提示, 该种免疫策略能够打破机 体对自身分子的免疫耐受，并且在肿瘤免疫治疗领域 中建立了一个综合治疗的新模式.

Niethammer等人 ${ }^{[40]}$ 制备了以减毒的鼠伤寒沙门 氏菌为载体的VEGFR-2 DNA疫苗, 该疫苗在小鼠体 内能够产生保护性的抗肿瘤免疫反应和治疗性的抗 肿瘤转移免疫反应，体外 $\mathrm{C}_{\mathrm{r}} 51$ 释放实验显示，免疫小 鼠的脾细胞能够特异识别转染了小鼠VEGFR-2 的黑 色素瘤细胞. 免疫组织化学染色显示在免疫小鼠肿瘤 组织血管周围有 $\mathrm{CD} 8^{+} \mathrm{T}$ 淋巴细胞浸润。体内淋巴细 
胞亚群去除实验证实抗肿瘤免疫反应依赖于 $\mathrm{CD} 8^{+} \mathrm{T}$ 淋巴细胞, CTL介导的内皮细胞杀伤效应提示以减毒 的鼠伤寒沙门氏菌为载体的VEGFR-2 DNA疫苗打破 了机体对自身抗原VEGFR-2 的免疫耐受, 抑制了肿 瘤血管生成和肿瘤的生长.

碱性成纤维细胞生长因子(bFGF/FGF2) 是一种重 要的促血管生成因子, 它由肿瘤细胞、巨噬细胞产生 或由细胞外基质释放，并以自分泌或旁分泌的方式发 挥作用. 它能够上调一些重要的促血管生成因子如 VEGF以及纤溶酶原激活物，并通过Bcl-2 途径抑制内 皮细胞的调亡. bFGF主要通过与其高亲和性受体一 成纤维细胞生长因子 I 型受体(FGFR1)的结合来发挥 其生物活性作用 ${ }^{[41]}$. Plum等人 ${ }^{[42]}$ 研究发现, 合成的 FGF2 肝素结合结构域肽段和受体结合结构域肽段在 体外均能抑制人脐静脉内皮细胞的增殖, 随后将上述 两种肽段以脂质体为佐剂分别免疫小鼠，发现接受了 肝素结合结构域肽疫苗免疫的小鼠体内产生了针对 FGF2 的特异性抗体，该抗体能够抑制FGF2 与硫酸肝 素的结合, 抑制了明胶海绵模型的肿瘤血管生成, 并 在肿瘤转移模型中抑制了肿瘤的生长.

抗肿瘤血管生成主动免疫反应具有诸多优点. 首先, 主动免疫治疗给药方便, 无需长时间反复给药; 其次, 主动免疫依靠自身机体产生特异性的抗肿瘤体 液免疫反应或细胞免疫反应，相对外源性补充制剂 (如抗体、小分子化合物)其副作用较小，且抗肿瘤作 用持续时间较长; 再次, 主动免疫所需费用较低. 尽 管抗肿瘤血管生成主动免疫反应取得了较大的进展, 但是, 到目前为止, 该领域的研究几乎都处在临床前 期试验阶段, 在其进入临床试验之前, 仍需进行大量 的研究以解决一些目前面临的问题, 如抗原的特异 性、免疫反应强度的调控、优化免疫制剂剂型、减少 对正常生理性血管生成作用的影响等.

\section{3 展望}

自肿瘤的生长和转移依赖新生血管生成这一观 点提出以来, 基础研究、动物实验、临床前期试验以 及抗肿瘤血管生成单克隆抗体的成功研发和上市均 证实了上述观点, 并且使得抗肿瘤血管生成成为了一 种有效的治疗肿瘤的方法, 虽然较传统治疗其有诸多
优点, 但抗血管生成治疗仍有不足和缺陷. 首先, 肿 瘤生长早期, 当肿瘤直径小于 $2 \sim 3 \mathrm{~mm}$ 时, 肿瘤细胞 依靠单纯的被动扩散既可获得生长所需的氧和营养 成分 ${ }^{[1]}$, 因此，单纯的抗血管生成不能彻底根除肿瘤. 其次, 尽管目前抗血管治疗所选用的靶向较特异, 但 仍有一些潜在的副作用，如影响伤口愈合，影响女性 月经周期等，并且其在儿童肿瘤的应用上有局限性. 因此，随着分子生物学和免疫学的发展, 可通过多种 方法发现更多的肿瘤特异性内皮细胞标志，如基因表 达系列分析的方法(SAGE)分析正常内皮细胞和增殖 内皮细胞的基因表达、噬菌体展示技术发现 新的内 皮细胞受体、蛋白组学的方法发现调节内皮 细胞生 长的肽段或蛋白质等 ${ }^{[43]}$. 再者, 由于肿瘤的发生和发 展是一个非常复杂的过程, 受诸多因素调控, 任何一 种单一的治疗方法都不可能彻底根除肿瘤, 将抗血管 生成治疗与传统的肿瘤治疗方法相结合应当成为一 种新的肿瘤综合治疗模式.

\section{参考文献}

1 Folkman J. Tumor angiogenesis: therapeutic implications. N Engl J Med, 1971, 285: 1182 1186

2 Frank A S. Mechanisms and future directions for angiogenesisbased cancer therapies. J Clin Oncol, 2002, 20: 3906 3927[DOI]

3 Kerbel R S, Folkman J. Clinical translation of angiogenesis inhibitors. Nat Rev Cancer, 2002, 2: 727 739 [DOI]

4 Zetter B R. Angiogenesis and tumor metastasis. Annu Rev Med, 1998, 49: 407 424[DOI]

5 Boehm T, Folkman J, Browder T, et al. Anti-angiogenic therapy of experimental cancer does not induce acquired drug resistence. Nature, 1997, 390: 404 407 [DOI]

6 Folkman J. Angiogenesis in cancer, vascular, rheumatoid and other diseases. Nature Med, 1995, 1: 27 31

7 Carmeliet P, Jain R K. Angiogenesis in cancer and other diseases. Nature, 2000, 407: 249 257[DOI]

8 Kim K J, Li B, Winer J, et al. Inhibition of vascular endothelial growth factor-induced angiogenesis suppresses tumour growth in vivo. Nature, 1993, 362(6423): 841 844[DOI]

9 Takayama K, Hikaru U, Yoichi N, et al. Suppression of tumor angiogenesis and growth by gene transfer of a soluble form of vascular endothelial growth factor receptor into a remote organ. Cancer Res, 2000, 60: 2169 2177

10 Lin P L, Jake A B, Ann A, et al. Antiangiogenic gene therapy targeting the endothelium-specific receptor tyrosine kinase Tie2. Proc Natl Acad Sci USA, 1998, 95: 8829 8834[DOI]

11 Ferrara N, Terri D S. The biology of vascular endothelial growth factor. Endocr Rev, 1997, 18: 4 25[DOI] 
12 Preweet M, James H, Li Y W, et al. Antivascular endothelial growth factor receptor (Fetal Liver Kinase 1)monoclonal antibody inhibits tumor angiogenesis and growth of several mouse and human tumors. Cancer Res, 1999, 59: 5209 5218

13 Niederman T M J, Ghogawala Z, Carter B S, et al. Antitumor activity of cytotoxic $\mathrm{T}$ lymphocytes engineered to target vascular endothelial growth factor receptors. Proc Natl Acad Sci USA, 2002, 99(10): 7009 7014[DOI]

14 Hackel P O, Zwick E, Prenzel N, et al. Epidermal growth factor receptors: critical mediators of multiple receptor pathways. Curr Opin Cell Biol, 1999, 11: 184 189[DOI]

15 Gleave M E, Hsieh J T, Wu H C, et al. Epidermal growth factor receptor-mediated autocrine and paracrine stimulation of human transitional cell carcinoma. Cancer Res, 1993, 53: 5300 5307

16 Takashi K, Paul S, Joel W S, et al. Inhibition of angiogenesis by the antiepidermal growth factor receptor antibody ImClone C225 in androgen-independent prostate cancer growing orthotopically in nude mice. Clini Cancer Res, 2002, 8: 1253 1264

17 Brooks P C, Stromblad S, Klemke R, et al. Anti-integrin $\alpha v \beta 3$ blocks human breast cancer growth and angiogenesis in human skin. J Clin Invest, 1995, 96(4): 1815 1822

18 Friedlander M, Brooks P C, Shaffer R W, et al. Definition of two angiogenic pathways by distinct alpha $\mathrm{v}$ integrins. Science, 1995, 270(5241): 1500 1502

19 Brooks P C, Stromblad S, Sanders L C, et al. Localization of matrix metalloproteinase MMP-2 to the surface of invasive cells by interaction with integrin $\alpha v \beta 3$. Cell, 1996, 85(5): 683 693[DOI]

20 John C G, Thomas N C, Paul R P, et al. Targeted antiangiogenic therapy for cancer using Vitaxin: a humanized monoclonal antibody to the integrin $\alpha v \beta 3$. Clin Cancer Res, 2000, 6: 3056 3061

21 Aberle H, Schwartz H, Kemler R. Cadherin-catenin complex: protein interactions and their implications for cadherin function. $\mathrm{J}$ Cell Biochem, 1996, 61: 514 523[DOI]

22 Liao F, Li Y, O'Connor W, et al. Monoclonal antibody to vascular endothelial (VE)-cadherin is a potent inhibitor of angiogenesis, tumor growth and metastasis. Cancer Res, 2000, 60: 6805 6810

23 Monica C, Lucia Z, Fabrizio O, et al. A monoclonal antibody to vascular endothelial-cadherin inhibits tumor angiogenesis without side effects on endothelial permeability. Blood, 2002, 100: 905 911 [DOI]

24 Rosenberg S A. Progress in human tumor immunology and immunotherapy. Nature, 2001, 411: 380 384[DOI]

25 Karlsen A E, Dyrbert T. Molecular mimicry between non-self, modified self and self in autoimmunity. Semin Immunol, 1998, 10: 25 34[DOI]

26 Wei Y Q, Wang Q R, Zhao X, et al. Immunotherapy of tumors with xenogeneic endothelial cells as a vaccine. Nat Med, 2000, 6: 1160 1166[DOI]

27 Wei Y Q, Huang M J, Yang L, et al. Immunogene therapy of tumors with vaccine based on xenopus homologous vascular endothelial growth factor as a model antigen. Proc Natl Acad Sci USA, 2001, 98: 11545 11550[DOI]

28 He Q M, Wei Y Q, Tian L, et al. Inhibition of tumor growth with a vaccine based on xenogeneic homologous fibroblast growth factor receptor-1 in mice. J Biol Chem, 2003, 278(24): 21831 21836 [DOI]

29 Lou Y Y, Wei Y Q, Yang L, et al. Immunogene therapy of tumor with vaccine based on the ligand binding domain of chick homologous integrin $\beta_{3}$. Immunol Invest, 2002, 31: 51 69[DOI]

30 Su J M, Wei Y Q, Tian L, et al. Active immunogene therapy of cancer with vaccine on the basis of chicken homologous matrix metalloproteinase-2. Cancer Res, 2003, 63: 600 607

31 Liu J Y, Wei Y Q, Yang L, et al. Immunotherapy of tumors with vaccine based on quail homologous vascular endothelial growth factor receptor-2. Blood, 2003, 102: 1815 1823[DOI]

32 Lu Y, Wei Y Q, Tian L, et al. Immunogene therapy of tumors with vaccine based on xenogeneic epidermal growth factor receptor. J Immunol, 2003, 70(6): 3162 3170

33 Overwijk W W, Lee D S, Surman D R, et al. Vaccination with a recombinant vaccinia virus encoding a "self" antigen induces autoimmune vitiligo and tumor cell destruction in mice: requirement for CD4(+) T lymphocytes. Proc Natl Acad Sci USA, 1999, 96: 2982 2987[DOI]

34 Toes R E M, Ossendrop F, Offringa R, et al. CD4 ${ }^{+}$T cells and their role in antitumor immune responses. J Exp Med, 1998, 189: 753 756[DOI]

35 Pardoll D M. Inducing autoimmune disease to treat cancer. Proc Natl Acad Sci USA, 1999, 96: 5340 5342[DOI]

36 De Silva H D, Van Driel I R, La Gruta N, et al. CD4 ${ }^{+} \mathrm{T}$ cells, but not $\mathrm{CD}^{+} \mathrm{T}$ cells, are required for the development of experimental autoimmune gastritis. Immunology, 1998, 93: 405 408[DOI]

37 Kumar V, Stellrecht K, Sercarz E. Inactivation of T cell receptor peptide-specific CD4 regulatory $\mathrm{T}$ cells induces chronic experimental autoimmune encephalomyelitis (EAE). J Exp Med, 1996 , 184: $1609 \sim 1617$

38 Li Y W, Wang M N, Li H L, et al. Active immunization against the vascular endothelial growth factor receptor flk1 inhibits tumor angiogenesis and metastasis. J Exp Med, 2002, 195: 1575 1584 [DOI]

39 Nair S, Boczkowski D, Moeller B, et al. Synergy between tumor immunotherapy and anti-angiogenic therapy. Blood, 2003, 102: 964 971[DOI]

40 Niethammer A G, Xiang R, Becker J C, et al. A DNA vaccine against VEGF receptor 2 prevents effective angiogenesis and inhibits tumor growth. Nature Med, 2002, 8: 1369 1375[DOI]

41 Martin Hagedornm Andreas Bikfalvi. Target molecules for antiangiogenic therapy: from basic research to clinical trials. Crit Rev Oncol: Hematol, 2000, 34: 89 110

42 Plum S M, Holaday J W, Ruiz A, et al. Administration of a liposomal FGF-2 peptide vaccine leads to abrogation of FGF-2- mediated angiogenesis and tumor development. Vaccine, 2001, 19: 1294 1303[DOI]

43 Frank A S. The therapeutic potential of novel antiangiogenic therapies. Expert Opin Investig Drugs, 2003, 6: 923 932 